\title{
Initial Presentation of HIV Infection With Two Successive Acute Arterial Thromboses: A Case Report
}

\author{
Bizhan Ziaian ${ }^{1}$; Sam Moslemi ${ }^{1, *}$; Maryam Tahamtan $^{2}$ \\ ${ }^{1}$ Colorectal Research Center, Shiraz University of Medical Sciences, Shiraz, IR Iran \\ ${ }^{2}$ Department of Cardiology, Shiraz University of Medical Sciences, Shiraz, IR Iran \\ *Corresponding Author: Sam Moslemi, Colorectal Research Center, Shiraz University of Medical Sciences, Shiraz, IR Iran. Tel:+98-7132306972, E-mail: moslemis@sums.ac.ir
}

Received: January 28, 2013; Revised: November 16, 2014; Accepted: December 10, 2013

\begin{abstract}
Introduction: One of the complications of HIV infection is greater risk of thromboembolic events. A variety of mechanisms has been found to be responsible for prothrombotic tendency in patients with HIV infection.

Case Presentation: A 27-year-old heterosexual smoker man was referred to our center due to a sudden-onset severe left lower extremity pain and claudication since three days prior to admission. In physical examination, end extremity coldness and discoloration as well as left lower extremity pulselessness were found. Color-Doppler sonography revealed a large thrombus in the left common iliac artery and two thrombi in the distal halves of both anterior and posterior tibialis arteries, so the patient was transferred to the operating room for proximal thrombectomy where the blood flow was reestablished and all pulses were then detectable. Two days later, the patient developed another similar episode from knee down and underwent the second thrombectomy. In evaluation, HIV Ab had positive result by ELISA. Conclusions: This case inspires consideration of HIV infection as a leading cause of thromboembolic event in individuals affected by the first episode of unprovoked one in whom diagnosis of HIV infection has not been established yet.
\end{abstract}

Keywords:Thrombosis; HIV Infections; Initial Presentation

\section{Introduction}

Acute ischemia of an extremity as a consequence of arterial occlusion caused by a superimposed thrombosis on an atherosclerotic plaque or an embolus contributes to reduced blood supply to muscle, nerve, subcutaneous tissue and skin (1). One of the complications of HIV infection is greater risk of thromboembolic events. Many mechanisms have been found to be responsible for prothrombotic tendency in patients with HIV infection (2). Although, thrombosis is mostly found in veins, the number of cases with arterial thrombosis is rapidly growing (3). However, occurrence of arterial thrombosis as the first presentation of HIV infection is extremely rare; reviewing the literature revealed only two previous case reports $(4,5)$. We presented a 27 -year-old man with two successive acute arterial thromboses as the initial presentation of HIV infection.

\section{Case Presentation}

A 27-year-old man from Yasuj (central part of Iran) was hospitalized from 5th to 18th of March 2012 in surgery ward of Nemazee Teaching Hospital, Shiraz University of Medical Sciences, Shiraz, Iran, due to intermittent episodes of acute lower extremity arterial thrombosis. Symptoms initiated three days prior to admission with a severe abrupt-onset entire left lower extremity pain and claudication not relieved by analgesics. He had a 10 pack- year history of cigarette smoking and a multipartner sexual relationship. Otherwise, his medical history showed a healthy young man up to the present illness. The patient's family history was unremarkable for thrombophilia. In physical examination, he was afebrile with a good general appearance and his blood pressure, pulse rate and respiratory rate were $120 / 80 \mathrm{mmHg}, 100 \mathrm{bpm}$ and $16 / \mathrm{min}$, respectively. His left lower extremity was cold from knee down and bluish discoloration of digits was noted.

Left femoral artery pulse was undetectable as well as its subsequent branches. Sensory and motor functions of the entire left lower extremity were intact. Other parts of physical examination had normal findings except for an oral aphthous lesion and a healing genital ulcer with a negative Pathergy test. Color-Doppler sonography of the left lower extremity arteries revealed a thrombus in the left common iliac artery, $5 \mathrm{~cm}$ in length, initiating from the abdominal aorta bifurcation. Trivial blood flow in the common iliac artery and decreased blood flow in the lower branches were found, so that the blood flow velocity was $27 \mathrm{~cm} / \mathrm{sec}$ in the left common femoral artery compared to $58 \mathrm{~cm} / \mathrm{sec}$ velocity of the normal opposite side. Evaluation of distal arteries including anterior and posterior tibialis arteries demonstrated a complete thrombosis in the lower halves of both with no blood flow. Anticoagulant therapy was promptly initiated and

Copyright (C) 2014, Iranian Red Crescent Medical Journal. This is an open-access article distributed under the terms of the Creative Commons Attribution-NonCommercial 4.0 International License (http://creativecommons.org/licenses/by-nc/4.0/) which permits copy and redistribute the material just in noncommercial usages, provided the original work is properly cited. 
Fogarty catheter was inserted for proximal thrombectomy. After the operation, the patient's end extremity was warm and he felt no pain anymore. Weak pulses of both dorsalis pedis and posterior tibialis arteries were also detectable. Forty-eight hours later, the patient developed another episode of cold end extremity and pulselessness in the same limb. In color-doppler sonography, despite establishment of triphasic blood flow of the left lower extremity arteries up to midpart of distal arteries, no flow was detected in the distal halves of anterior and posterior tibialis arteries due to an embolus/thrombus. Therefore, the patient underwent Fogarty insertion for thrombectomy again. After the second surgery, all previous symptoms and signs were removed and follow-up CT-angiography revealed normal course and caliber of all the arteries of the left lower extremity with no evidence of collateral formation in favor of chronic vascular insufficiency. Meanwhile, to find out the reason of recurrent thromboembolic events in an otherwise healthy young man, an extensive work-up was performed. Lab investigation revealed a CBC with $\mathrm{Hg}$ of $16.7 \mathrm{~g} / \mathrm{dL}, \mathrm{WBC}$ of $7000 /$ $\mathrm{mm}^{3}$ and platelet count of $179000 / \mathrm{mm}^{3}$. PT, INR and PTT were 13 seconds, 1 and 25 seconds, respectively. Lipid profile showed total cholesterol of $202 \mathrm{gr} / \mathrm{dL}$, TG of $146 \mathrm{gr} / \mathrm{dL}$, HDL-c of $37 \mathrm{gr} / \mathrm{dL}$ and LDL-c of $136 \mathrm{gr} / \mathrm{dL}$. Serum levels of antiphospholipid-antibodies, anticardiolipin antibody (ACLA), P- and C-ANCA and anti- $\beta 2$ glycoprotein IgM and IgG were all within the normal ranges. Liver function test showed no abnormality and viral markers for hepatitis $\mathrm{B}$ and $\mathrm{C}$ had negative results. Homocysteine level was normal and LDH was $445 \mathrm{IU} / \mathrm{L}$. Coagulation factor assay revealed no abnormal findings and results were not in favor of protein $\mathrm{C} / \mathrm{S}$ or antithrombin deficiency. Investigations showed no factor $\mathrm{V}$ Leiden gene mutation. Other blood tests had normal findings. Regarding proximal involvement of lower extremity arteries, abdomen pelvic sonography, helical chest and abdomenopelvic CT-scan demonstrated no mass, organomegally or the third space fluid collection. EKG (electrocardiography) and trans esophageal echocardiography (TEE) excluded any cardiogenic source of emboli. Ultimately, we found a reactive HIV Ab ELISA with the same result in repetition. Western blot test confirmed the diagnosis of HIV infection. The patient was then discharged with antiplatelet and anticoagulant and referred to a HIV center to receive medical support. In outpatient follow-up, endogenous anticoagulants were rechecked two weeks after the acute phase with all results within the normal limits.

\section{Discussion}

Patients with HIV infection face with a 4 to 6-fold higher risk of thromboembolic events with an incidence ranging from $0.26 \%$ to $7.6 \%(2,6)$. Despite the absence of largecohort studies to determine the precise incidence of arterial thrombosis in HIV infection, it was estimated as $0.45 \%$ in a small-cohort study (7). Acute arterial thrombosis as an initial presentation of HIV infection was reported in two previous cases. Witz et al. presented a 53-year-old heterosexual man with an acute brachial artery thrombosis whose coagulation factor assay showed no abnormality. Further evaluation ascertained HIV infection in patient with a CD4 count of 112 (4). Another case was a 42-year-old woman with an acute dorsalis pedis arterial thrombosis whose work-up results revealed protein $\mathrm{S}$ deficiency, hepatitis C and HIV-1 infection (5), although not clarified sufficiently yet, different potential mechanisms account for prothrombosis and hypercoagulation in HIV infection. These include the presence of antiphospholipid-antibodies, lower activities of endogenous anticoagulants such as protein $C$, protein $S$ and antithrombin III, increased platelet activity and excess risk of endothelial dysfunction in patients with HIV infection (2). It is believed that higher incidence of thromboembolism in patients with HIV-infection is associated with metabolic abnormalities such as insulin resistance, fat redistribution and increased atherogenic cholesterol and triglyceride levels, which is caused by advent of highly active antiretroviral therapy (HAART ) (8). The key event in the initiation and progression of atherosclerosis is endothelial dysfunction. Accelerated endothelial activation could be due to either viral infection itself because of direct impact of viral components including gp120 and TAT on the endothelium or a side effect of HAART (9). Endothelial activation leads to increased expression of adhesion molecules such as intercellular adhesion molecule (ICAM)-1 and endothelial adhesion molecule (E-selectin). Serum levels of inflammatory cytokines such as tumor necrosis factoralpha (TNF- $\alpha$ ) and interleukin-6 (IL-6) are also increased (10). Satchell CS et al. found that platelets of patients with HIV infection were more reactive to platelet agonists such as epinephrine compared to less platelet aggregation in non-infected patients. It could be due to multiple underlying defects in platelet function in HIV infection (11). Due to the association of HIV infection and autoimmune disorders such as antiphospholipid antibody syndrome, evaluation of serum level of antiphospholipidantibodies such as anticardiolipin in patients with HIV infection has been introduced as a fundamental step in the management of both symptomatic and asymptomatic patients (11). Frequency of thrombophilic abnormalities in HIV infection increases with its progression to AIDS and correlates with the severity of immunosuppression measured by the CD4 cell counts, also with the presence of concurrent opportunistic infections or neoplastic processes $(12,13)$. In our case, an extensive evaluation was performed due to the first unprovoked thromboembolic event. We were concerned of the Burger's disease because of the patient's medical history remarkable for smoking. However, a normal CT-angiography excluded our impression. We suppose that the second episode of the left lower extremity ischemia could be due to an embolus lodged in the midpart of distal arteries because of probable floating thrombus particles originated from the first Fogarty 
insertion. However, it could be due to the rupture or erosion of atherothrombotic plaques in the distal halves of anterior and posterior tibialis arteries previously reported in color doppler sonography by means of a sudden onset of post-thrombectomy blood flow reestablishment of the common iliac artery resulting to an acute superimposed thrombosis. We found no other underlying disorder responsible for the current presentation except HIV infection.

Acute arterial thromboembolism as an initial presentation of HIV infection is extremely rare. However, as it can occur via multiple potential mechanisms in the course of disease, it could introduce a new troublesome condition. Therefore, we should consider HIV Ab evaluation for any unprovoked thromboembolic event, especially in an otherwise healthy youth.

\section{References}

1. O'Donnell TF, Jr.. Arterial diagnosis and management of acute thrombosis of the lower extremity. Can J Surg. 1993;36(4):349-53.

2. Shen YM, Frenkel EP. Thrombosis and a hypercoagulable state in HIV-infected patients. Clin Appl Thromb Hemost. 2004;10(3):27780.

3. Robbs JV, Paruk N. Management of HIV vasculopathy - a South African experience. Eur J Vasc Endovasc Surg. 2010;39 Suppl 1:S25-31.

4. Witz M, Lehmann J, Korzets Z. Acute brachial artery thrombosis as the initial manifestation of human immunodeficiency virus infection. Am J Hematol. 2000;64(2):137-9.

5. Archer KA, Lembo TF, Jr., Haber JA. Protein S deficiency and lowerextremity arterial thrombosis: complicating a common presentation. J Am Podiatr Med Assoc. 2007;97(2):151-5.

6. Tichelaar VY, Sprenger HG, Lijfering WM. Risk of Venous and Arterial Thrombosis in HIV-infected Patients: A Narrative Review. J Coagul Disord. 2007.

7. Lijfering WM, Ten Kate MK, Sprenger HG, van der Meer J. Absolute risk of venous and arterial thrombosis in HIV-infected patients and effects of combination antiretroviral therapy. J Thromb Haemost. 2006;4(9):1928-30.

8. Mondy KE. Determinants of endothelial function in human immunodeficiency virus infection: a complex interplay among therapy, disease, and host factors. J Cardiometab Syndr. 2008;3(2):88-92.

9. Hurlimann D, Weber R, Enseleit F, Luscher TF. [HIV infection, antiretroviral therapy, and endothelium]. Herz. 2005;30(6):472-80.

10. Mu H, Chai H, Lin PH, Yao Q, Chen C. Current update on HIV-associated vascular disease and endothelial dysfunction. World J Surg. 2007;31(4):632-43.

11. Satchell CS, Cotter AG, O'Connor EF, Peace AJ, Tedesco AF, Clare A, et al. Platelet function and HIV: a case-control study. AIDS. 2010;24(5):649-57.

12. Giordano N, Amendola A, Papakostas P, Cipolli F, Agate VM Martini G, et al. Possible pathogenetic role of antiphospholipid antibodies in a clinical case of human immunodeficiency virus infection with peripheral polyneuropathy and arterial thrombosis. New Microbiol. 2005;28(3):261-3.

13. Saif MW, Greenberg B. HIV and thrombosis: a review. AIDS Patient Care STDS. 2001;15(1):15-24. 